\title{
Strain rate jump tests on an austenitic stainless steel with a modified tensile Hopkinson split bar
}

\author{
Naiara I. Vazquez Fernandez ${ }^{1, *}$, Matti Isakov $^{1,2}$, Mikko Hokka $^{1}$, and Veli-Tapani Kuokkala ${ }^{1}$ \\ ${ }^{1}$ Tampere University of Technology, Laboratory of Materials Science, Korkeakoulunkatu 6, P.O.Box 589, FI-33101, Finland \\ ${ }^{2}$ Materials and Simulation Methods, Fraunhofer Institute for High-Speed Dynamics, Ernst-Mach-Institut, Eckerstrasse 4, \\ 79104 Freiburg, Germany
}

\begin{abstract}
This paper presents an improved experimental setup for high strain rate testing based on the modified Tensile Hopkinson Split Bar device developed previously at TUT. The test setup can be used to study the effects of a sudden large change in the strain rate on the stress flow of the material. The setup allows deforming the sample at a low rate and at isothermal conditions before the high rate loading. During the strain rate jump, the deformation rate is rapidly increased by approximately six orders of magnitude. In this work, the low and high rate deformation of the specimen was recorded with a combination of low and high-speed digital cameras and analyzed using the Digital Image Correlation technique. The measurement provides information about the effects of the strain rate jump on the macroscopic response of the material and allows accurate observation of the deformation of the sample just before, during, and immediately after the strain rate jump, when the conditions change from isothermal to adiabatic. In this paper, we present the results for a metastable austenitic stainless steel and discuss the effects of the strain rate jump on the strain-hardening rate, compare the experimental results with numerical results from a thermomechanical model, and evaluate the effects of the preceding deformation at a low strain rate on the strain localization. We conclude that the strain rate jump results in a clear decrease in the strain-hardening rate, the deformation following the jump is uniform along the gauge section, and that the strain localization is not significantly affected by the strain rate or the amount of pre-strain in the studied conditions.
\end{abstract}

\section{Introduction}

Various requirements set by the modern society demand the steel industry to improve the properties of steels. This includes higher ductility and stronger strain hardening capability. However, these goals are difficult to reach with simple materials and microstructures, and structures that are more complex are therefore being developed. For example, the metastable austenitic stainless steels produce a good combination of ductility and strength with the help of martensitic phase transformation. The phase transformation has a strong effect on the mechanical properties of the steels, but it also increases the material's temperature and strain rate sensitivities.

Many scientists [1-5] have focused their efforts on explaining the effects of the martensitic phase transformation on the resulting microstructure and mechanical properties at a wide range of strain rates. The strain rate, temperature, and adiabatic heating influence the active microplastic mechanisms that govern the overall mechanical behavior and plasticity of the steel. Higher strain rates typically slow down the formation of martensite. In the literature [6-10], this behavior is typically explained by the adiabatic heating of the material at higher strain rates. Most of the recent work involves testing only at constant strain rates and studying the mechanical properties and microstructural changes only after the deformation. In contrast, Ghosh [11], and Klepaczko and Chiem [12] highlighted the importance of the deformation history on the material response. In general, the overall strain rate sensitivity includes both the instantaneous and the evolutionary effects of the loading rate on the material behavior. Materials undergoing a phase transformation have a microstructure that evolves considerably during plastic deformation, and therefore it is crucial to distinguish between the two above mentioned loading rate effects. Following this approach, Isakov et al. [13] performed strain rate jump experiments on a metastable austenitic stainless steel, and observed that both the strain-hardening rate and the phase transformation rate decreased instantaneously after the jump. Furthermore, a monotonous low strain rate test revealed a notable increase in the strain-hardening rate at around 0.15 of true strain, in comparison with the high strain rate test, where the strain-hardening rate was more or less constant with respect to the amount of strain. The martensitic phase transformations occurring after 0.15 of

\footnotetext{
Corresponding author: naiara.vazquez@tut.fi
} 


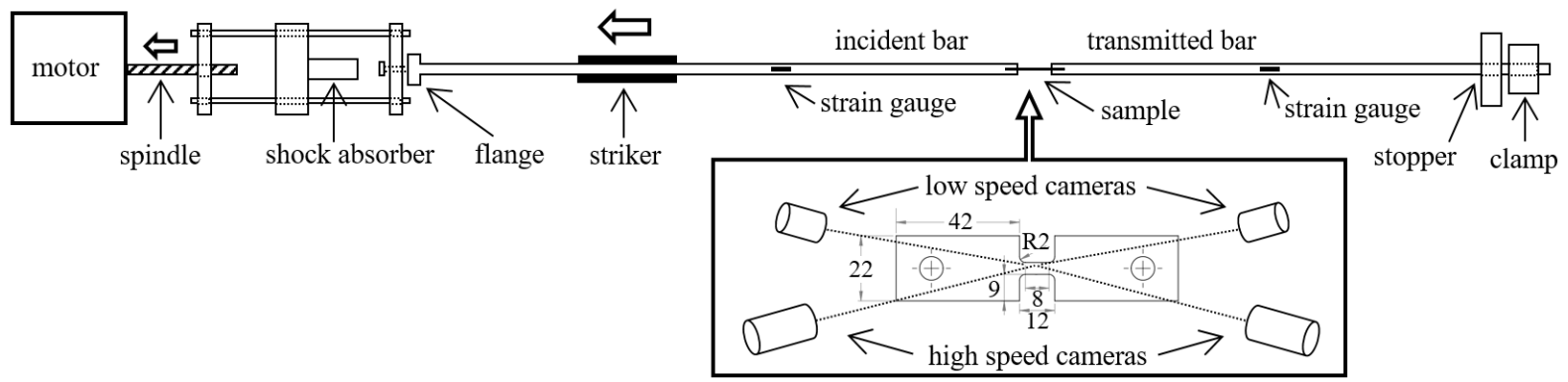

Fig. 1. A schematic picture of the experimental setup.

true strain become crucial for the material hardening. Thus, analyzing the material behavior around that amount of strain can reveal meaningful information regarding the martensitic phase transformation.

Isakov et al. [14] also developed a phenomenological material model that describes the strain rate dependent plasticity of this material. This model predicts the flow stress of the material while the deformation is uniform, i.e., until the strain starts to localize. The strain localization and neck formation are also interesting features of the material behavior. However, the evaluation of post necking behavior is difficult since the simple equations for uniaxial stress-strain testing are no longer valid. The equations assume that the specimen gauge section is deforming uniformly and under onedimensional stress state, which no longer holds when the specimen necks. Furthermore, localized deformation within the neck cannot be easily measured with traditional techniques. Digital Image Correlation allows overcoming some of those issues. This technique is becoming a standard practice in experimental mechanics, and it enables the evaluation of the full field deformation and strains on the sample surface during the entire test, including the neck formation.

This work aims to deepen the understanding of the effects of strain rate on the martensitic phase transformation and the overall mechanical behavior of metastable austenitic stainless steels. Tensile tests were performed at both constant strain rate and with a sudden increase of strain rate after a certain pre-strain. The monotonous low strain rate results show an increase in the strain-hardening rate at around 0.15 true strain that does not occur in the monotonous high strain rate tests. The increase in the strain-hardening rate is caused by the martensitic phase transformation. Based on these results, the strain rate jumps were performed at the pre-strains of 0.10 and 0.23 of true strain, corresponding to the beginning and the end of the strong phase transformation period observed in the low rate results.

\section{Experiments and methods}

\subsection{Material}

The designation of the material tested in this work is EN 1.4318-2B, which is a metastable austenitic stainless steel provided by Outokumpu Stainless LTD (composition: $\mathrm{Cr}=17.4 \%, \mathrm{Ni}=6.7 \%, \mathrm{Mn}=1.18 \%$,
$\mathrm{Si}=0.38 \%, \mathrm{~N}=0.151 \%, \mathrm{C}=0.022 \%, \mathrm{Fe}=$ bal. $).$ The samples were laser-cut from a $2 \mathrm{~mm}$ thick sheet. The width of the gauge section of the specimens was $4 \mathrm{~mm}$, the length was $8 \mathrm{~mm}$, and the fillet or shoulder radius was $2 \mathrm{~mm}$. The speckle patterns for the digital image correlation were painted with a permanent marker directly on the sample surface without using any base coat.

\subsection{Testing equipment}

The tests were performed using the modified Tensile Hopkinson Split Bar (TSHB) device previously developed at Tampere University of Technology. The setup comprises a tension SHB device combined with a low speed screw-driven actuator device. An electric motor pulls the incident bar at a constant speed, while the far end of the transmitted bar is clamped. Thus, the sample can be deformed at a low strain rate $\left(\sim 2 \times 10^{-4}\right.$ $\left.\mathrm{s}^{-1}\right)$ before the high strain rate loading $\left(\sim 10^{3} \mathrm{~s}^{-1}\right)$. The high strain rate part of the test is carried out normally at any desired moment by impacting a striker tube against the flange machined at the end of the incident bar. Figure 1 shows a schematic picture of the setup. For further details, see ref. [15].

The deformation of the sample was analyzed by two separate Digital Image Correlation setups. The low strain rate part of the test was observed by two 5 MPix E-lite cameras with $200 \mathrm{~mm}$ Nikon lenses, while the high strain rate part of the test was imaged using two Photron SA-X2 high speed cameras with $100 \mathrm{~mm}$ Tokina lenses. The images were recorded at $1 \mathrm{fps}$ to cover the low strain rate part of the loading, and at $180 \mathrm{kfps}$ to cover the high strain rate loading. The size of the obtained images during the low strain rate loading was $2456 \times 2058$ pixels, whereas significantly lower resolution images of $256 \times 120$ pixels were obtained during the high rate loading. The recorded images were analyzed using the LaVision StrainMaster (DaVis) 3D-DIC software. The low strain rate tests were analyzed using a subset size of 55 pixels and a step size of 27 pixels, whereas the high strain rate tests were analyzed with a subset size of 11 pixels and a step size of 5 pixels.

The strain was calculated by comparing the deformed images to the non-deformed images. The reference image was taken prior to loading of the sample with all four cameras.

A $5 \mathrm{~mm}$ virtual extensometer was used to obtain the engineering strain on the sample gauge section in the 
loading direction. The position of the extensometer becomes crucial for the samples involving a strain rate jump, as there are two sets of image data for the strain. Therefore, the virtual extensometer for the high speed DIC was placed as close to the same position as possible as in the low speed DIC reference image. As a result, both low and high rate data can be taken to refer to the same reference condition. Then, the engineering strains were converted to true strains for the low and the high strain rate parts of the test before combining the true strain and true stress data for the complete jump tests. The necking behavior was analyzed by evaluating the longitudinal strain along a horizontal line where the extensometers were placed.

\subsection{Material model}

The predictions of the model developed by Isakov et al. [14] were compared with the experimental results of the monotonous and jump test experiments. A short summary of the model is first presented in the following.

The model relies on both instantaneous and evolutionary effects contributing to the flow stress $\left(\sigma_{Y}\right)$ of the material as shown in Equation 1:

$$
\sigma_{Y}=\sigma_{t h}+\sigma_{a}
$$

The thermal component $\left(\sigma_{t h}\right)$ corresponds to the instantaneous strain rate and temperature sensitivities of the material, i.e., the response of the material to a sudden change of strain rate and/or temperature at a constant microstructure. The athermal component $\left(\sigma_{a}\right)$ describes the flow stress of the material at reference conditions, i.e., the material properties in the static regime. However, this flow stress component is also dependent on the strain rate and temperature through its evolution equations. Those equations reflect the temperature and strain rate dependence of the microstructure evolution during deformation. This is mathematically shown in Equations 2 and 3, where $f^{\alpha^{\prime}}$ is the volume fraction of martensite and $d \varepsilon_{p l}$ is the increment in plastic strain. The athermal component of the flow stress depends on an internal variable $\delta$ (Equation 2), which evolves during plastic deformation (Equation 3). This variable can be interpreted as the macroscopic representation of glideobstacles in the microstructure. The effect of the martensitic transformation is two-fold. Firstly, it contributes directly to the flow stress (Equation 2) via so-called dynamic softening effect [16], i.e., at low volume fractions the martensitic transformation may operate as an additional deformation mechanism. Secondly, the martensitic transformation contributes notably to the strain hardening of the material by affecting the internal variable $\delta$ by increasing the amount of glide-obstacles.

$$
\begin{gathered}
\sigma_{a}=\mathrm{f}\left(\delta, d f^{\alpha^{\prime}} / d \varepsilon_{p l}\right) \\
d \delta / d \varepsilon_{p l}=\mathrm{g}\left(\delta, f^{\alpha^{\prime}}, d f^{\alpha^{\prime}} / d \varepsilon_{p l}\right)
\end{gathered}
$$

Thus, the flow stress at a given value of plastic deformation is calculated by means of a few internal state variables that result from solving the thermomechanical problem, in which the strain-induced phase transformation is accounted for. The martensitic phase transformation is based on the model originally presented by Olson and Cohen [17], with an addition of direct strain rate sensitivity of the transformation [14].

It is important to note here that the model does not involve an explicit equation between the flow stress and the current value of plastic strain. Instead, this relationship is obtained implicitly by integrating the state variables over the plastic deformation path. In this context, the integration is carried out over a variable strain rate and temperature history. Therefore, the model is well suited for describing the strain rate jump experiments discussed in this paper.

Moreover, due to the strong temperature dependence of the martensitic transformation and the deformationinduced heating taking place at high strain rates, the model is thermo-mechanically coupled, i.e., one needs to solve the temperature of the deforming material. In general, this involves heat transfer calculations. In the cases studied in this paper, however, this challenge can be solved analytically by noting that during the low strain rate part of the test the conditions can be assumed fully isothermal, i.e., the temperature is constant. Since during the high rate loading the conditions are fully adiabatic, there is no heat transfer so the material temperature increases according to the heat release that takes place during the deformation. Therefore, the model predictions can be calculated with a simple numerical integration, in which the material temperature during high rate loading is analytically obtained between the integration steps. The integration is based on the plastic work and heat release from the martensite transformation that took place at the previous step. It should be noted that this calculation approach is only valid for strain rates in the fully isothermal and fully adiabatic regimes. At intermediate strain rates, one needs to use, for example, the Finite Element Method to solve the heat transfer between the specimen and its surroundings.

The calibration of the model involves measuring stress-strain curves in monotonous (constant strain rate) and strain rate jump tests, in the strain rate and temperature regimes of interest, including the quantification of the corresponding phase transformation. In this work, the experimental data is compared with numerical calculations based on the model and parameters published in reference [14] (parameter set "Batch A").

\section{Results and discussion}

Figure 2 presents both the experimental and numerical true stress-strain curves of all the tests. The Figure also shows the simulated stress-strain curves obtained with the material model. Fig. 2a shows the tests performed at constant strain rates of $2 \times 10^{-4} \mathrm{~s}^{-1}$ and $10^{3} \mathrm{~s}^{-1}$. The tests involving a sudden change of strain rate are shown in Fig. 2b. The low strain rate part of the jump tests was carried out at the strain rate of $2 \times 10^{-4} \mathrm{~s}^{-1}$ up to the deformation of 0.10 or 0.23 of true strain, where the 


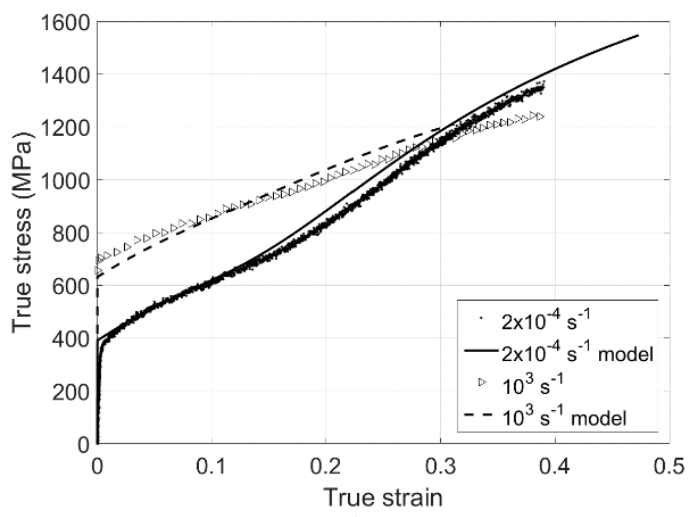

a)

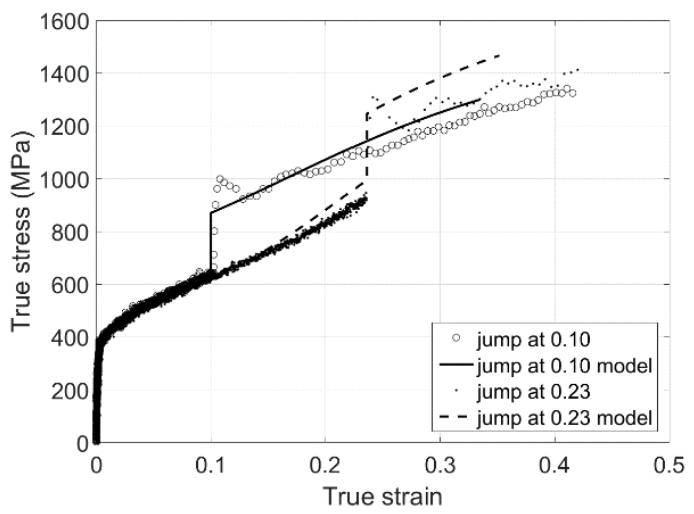

b)

Fig. 2. Experimental and numerical true stress vs. true strain curves for: a) monotonous strain rate tests at low and high strain rates and $b$ ) tests with a sudden increase of strain rate.

strain rate was suddenly increased to $10^{3} \mathrm{~s}^{-1}$. The quality of the stress data was not optimal due to artifacts introduced by the mechanical clamping of the specimen. Despite that, the trend of the results is still clearly visible. As can be seen, the numerical calculations from the model overlap the experimental data.

The monotonous curves presented above are associated with two different regimes of the mechanical behavior: the deformation at the lower strain rate corresponds to a quasi-static test where deformation takes place under isothermal conditions, whereas the deformation at the higher rate is adiabatic and occurs without heat transfer away from the sample. As can be seen in the curves in Fig. 2b, the change in the strain rate and the consequent change in the thermal conditions result in a change of the strain-hardening rate. As mentioned by Hecker et al. [6] and Talonen [8], the strong increase of the strain-hardening rate observed after approximately 0.15 low strain rate deformation can be explained by the martensitic phase transformation. In Fig. 2a, the slope of the stress vs. strain curve also changes significantly after approximately 0.15 of deformation. The adiabatic heating occurring at high strain rates reduces this effect, and typically $[6,8,9]$ the slope of the stress vs. strain curve decreases. In Fig. 2a, the strain-hardening rate of the material at the higher strain rate is significantly lower than at the lower strain rate. The difference is so significant that the flow stress of the material at large plastic strains in the low strain rate test is higher than in the high strain rate test. So, based on monotonous tests, the strain rate sensitivity of the steel actually becomes negative at higher strains.

The phase transformation rate has been observed to decrease immediately after a sudden increase of strain rate, leading to an immediate decrease in the strainhardening rate of the material $[13,14]$. To further study this effect, the strain rate jumps were performed at 0.10 and 0.23 of true strain, i.e., before and after the large change in the strain-hardening rate observed in the constant low strain rate test. The strain rate jump at 0.10 causes an immediate increase in the flow stress to an approximately same level as observed in the monotonous high strain rate test. In addition, the strain-hardening rate (slope of the stress-strain curve) also decreases immediately to a value close to that of the monotonous test. This behavior is expected since the microstructure at this stage is mostly austenitic regardless of the strain rate history $[8,9,13,14]$. In the jump test after a prestrain of 0.23 , the sudden increase of strain rate leads to a behavior similar to that observed in the jump after 0.10 . The flow stress increases, but the strain-hardening rate of the material right after the jump is similar to the strain-hardening rate observed in the monotonous high strain rate test. This suggests that the strain rate jump decreases the strain-hardening rate caused by the martensite formation by immediately decreasing the rate of further phase transformation.

Figure 3 shows waterfall plots of the true strain in the loading direction. Fig. 3a shows the sample in the nondeformed condition. In Figures $3 b$ to $3 e$, the legend shows the time corresponding to each profile. The strain profiles for the high strain rate part of a jump test start from the sudden increase of strain rate, and the low strain rate parts are not shown here for better visual readability of the Figure. The sign $\left({ }^{*}\right)$ indicates the moment at which the deformation is no longer uniform. The tests performed at a monotonous strain rate have a uniform deformation up to 0.39 true strain, while in the tests with a strain rate jump the strain localization starts at 0.42 true strain. The Figures show extensive deformation in the specimens outside the gage section of the specimens. This is a well-known fact typically observed for materials with a relatively low yield strength but a high strain-hardening rate. Inside the gage section, the deformation of the specimen is uniform until the onset of necking. It is very clear from the results that the sudden change of strain rate does not affect much the strain distribution along the gage section of the specimen, and the profiles obtained immediately after the jump are similar to those observed for the monotonous strain rate tests. The onset of plastic instability or necking seems also to be quite insensitive to the sudden change of strain rate. The uniform elongation does not change much even when the strain rate is increased during the test. The sizes of the necks or the width and overall shape of the strain profiles shown in Figure 3 are not significantly affected by the strain rate nor the sudden change of strain rate. 


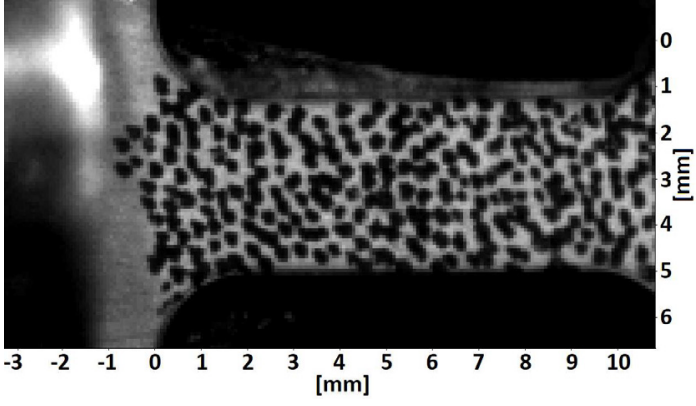

a)

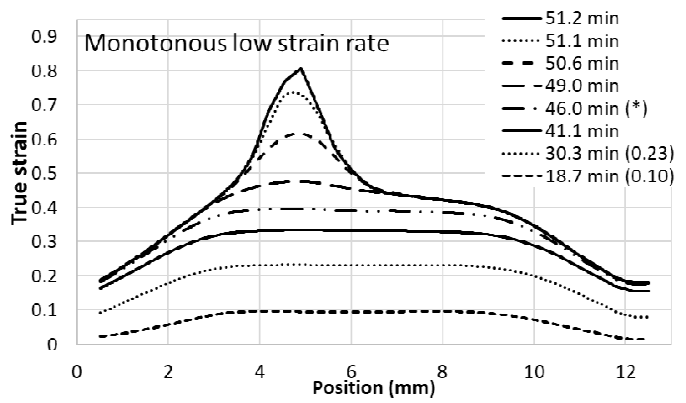

b)

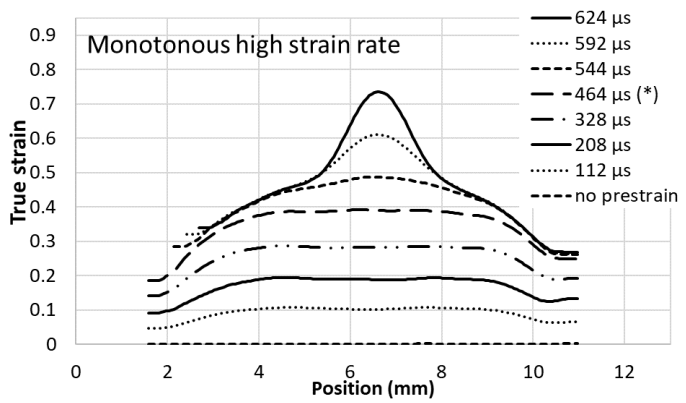

c)

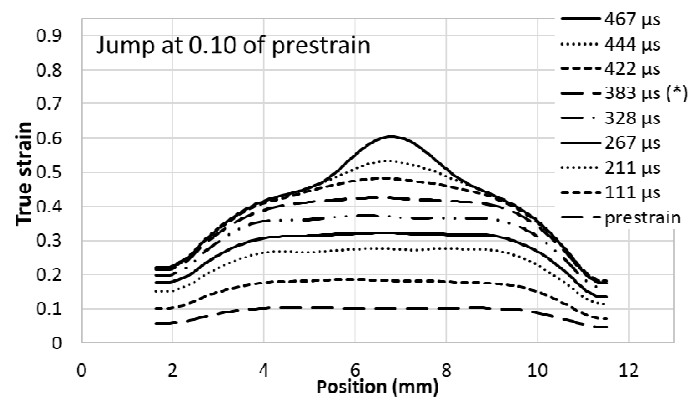

d)

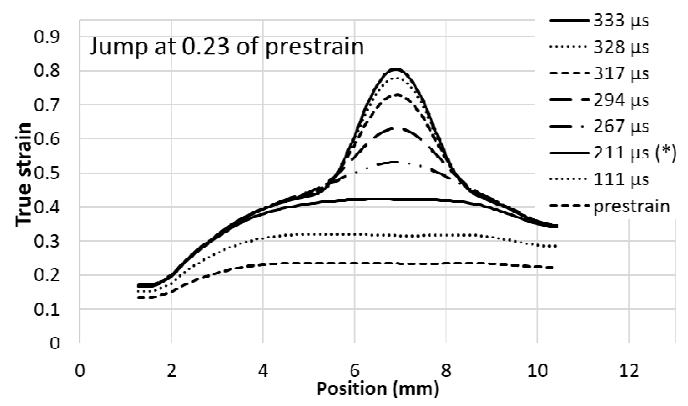

e)

Fig. 3. a) A high speed camera photograph of a non-deformed sample, b) waterfall plots showing axial true strain for the monotonous test at a low strain rate, c) monotonous test at a high strain rate, d) strain rate jump test at a pre-strain of 0.10 , and e) strain rate jump test at a pre-strain of 0.23 .
It is important to point out that only the sample with a pre-strain of 0.23 reaches failure with the first incident wave. The other samples, instead, break after consecutive reflections of the loading wave, and the analysis of strain is considered only until the end of the first incident wave. Consequently, the last profiles in Figures $3 \mathrm{c}$ and $3 \mathrm{~d}$ do not reflect the strain profile before the final failure but correspond simply to the strain at the moment when the incident pulse ends, and therefore, the exact comparison of the full localization behavior is difficult. Furthermore, more jump tests should be carried out to study also the sensitivity of the material to rapid changes in the strain rate at pre-strains close to 0.15 , where the martensitic transformation rate is highest.

\section{Concluding remarks}

In this work, a modified Tensile Split Hopkinson Pressure Bar device was used to study the effects of a sudden increase in the strain rate at different plastic strains on the mechanical behavior of a metastable austenitic stainless steel. The main findings of the study can be summarized as follows:

- The stress-strains curves for the jump tests show a clear effect of the sudden strain rate increase on the strain hardening behavior of the material.

- The previously published numerical model [14] can predict the results of the strain rate jump test realistically.

- The deformation of the sample remains uniform inside the gage section following the sudden increase of strain rate. This shows that the test method is feasible for analyzing material plasticity in tension, and especially for evaluating the instantaneous rate sensitivity of the material.

- The strain localization observed in these tests was not influenced much by the strain rate, the sudden change of strain rate, or the low strain rate deformation preceding the strain rate jump.

This work was funded by Academy of Finland under the grant number 294845.

\section{References}

1. D. Xu, J. Li, Q. Meng, Y. Liu, P. Li, J. Alloys and Compounds 614, 94-101 (2014)

2. J. Huang, X. Ye, Z. Xu, J. Iron and Steel Research, International 19 issue 10, 59-63 (2012)

3. C. Wang, H. Ding, M. Cai, B. Rolfe, Materials Science and Engineering: A 610, 65-75 (2014)

4. C. Wang, H. Ding, M. Cai, B. Rolfe, Materials Science and Engineering: A 610, 436-444 (2014)

5. A. Ma, A. Hartmaier, International Journal of plasticity 64, 40-55 (2015)

6. S.S. Hecker, M.G. Stout, K.P. Staudhammer, J.L. Smith, Metall. Trans. A 13, 619-626 (1982)

7. V. Talyan, R.H. Wagoner, J.K. Lee, Metall. Trans. A 29, 2161-2172 (1998) 
8. J. Talonen, Doctoral thesis, Helsinki University of Technology (2007)

9. J.A. Lichtenfeld, M.C. Mataya, C.J. Van Tyne, Metall. Trans. A 37, 147-161 (2006)

10. A. Andrade-Campos, F. Teixeira-Dias, U. Krupp, F. Barlat, E.F. Rauch, J.J. Gr Grácio, Strain 46, 283297 (2010)

11. A.K. Ghosh, Mater. Sci. Eng. A 463, 36-40 (2007)

12. J.R. Klepaczko, C.Y. Chiem, Mech. Phys. Solids 34, 29-54 (1986)

13. M. Isakov, S. Hiermaier, V.-T. Kuokkala, Metall. Mater. Trans. A 46, 2352-2355 (2015)

14. M. Isakov, M. May, S. Hiermaier, V.-T. Kuokkala, Materials and Design 106, 258-272 (2016)

15. M. Isakov, J. Kokkonen, K. Östman, V.-T. Kuokkala, Eur. Phys. J. Special Topics 225, 231242 (2016)

16. T. Narutani, G.B. Olson, M. Cohen, J. Phys. Colloq. 43, 429-434 (1982)

17. G.B. Olson, M. Cohen, Metall. Trans. A 6A, 791795 (1975) 